\title{
Shadow Effect Research in Tourism Development----- taking Lintong Jiang village site, Bian Que tomb and Lin Xiangru tomb for example
}

\author{
Li Hui \\ Xi'an University of Science and Technology, Xi'an,China,710054 \\ 616922788@qq.com
}

Keywords: Tourism development; Shadow effect; Jiang village site; Lin Xiangru tomb; Bian Que tomb

\begin{abstract}
Through the analysis of status quo, this paper points out shadow effect problems of the three major scenic spots: Lintong Jiang village sites, Bian Que tomb and Lin Xiangru tomb; makes in-depth analysis and mining of higher level scenic spot unique tourism value comparing with other places, such as unique health tourism resources of traditional Chinese medicine, neolithic culture including Longshan culture and Yangshao culture period and so on. Then makes in-depth analysis of the shadow effect causes, seeks the breakthrough strategy of shadow effect through comparative analysis of shadow area and being shadowed area, and finally puts forward integrated use strategies like filling vacancy method and avoiding hot spots, cooperation, and perfecting the existing tourist routes in order to break through shadow effect.
\end{abstract}

\section{Introduction}

Tourism shadow is also called destination image cover, that is, in a certain area of tourism destination, high level tourism resources, prominent features, large tourist destination product brand effect will be more outstanding in terms of tourism image, and have cover effect on other tourist destinations' image formation. In recent years, tourism becomes a significant new growth point of national economy, and as a world famous tourist hot spot, Shanxi Lintong county has 2 national 5 A grade scenic spots: museum of Qin Shihuang terracotta warriors, HuaQing pool scenic area and two national 4 A level scenic spots: Mount Li national forest park, Banpo Museum. Because of the famous scenic spots, in 2013, Lintong tourist visitors reached 5.38 million, 31.29\% up from a year earlier, and comprehensive tourism income 1.882 billion yuan, 31.22\% up from a year earlier. Because of the museum of Qin terra-cotta warriors and other scenic spots have been developed for a long time, and have long been known to tourists, they set up a clear image for tourists, and become Xian and Shanxi business card. Terra Cotta Warriors received up to 4.675 million people in 2013, 2.6 million HuaQing pool, Mount Li 980000, etc. Other tourist attractions, for example, Bian Que tomb, about $8 \mathrm{~km}$ away from Qin Terra Cotta Warriors , Lin Xiangru tomb, $15 \mathrm{~km}$ away in the east of Lintong and Jiang village site at the north shore of Lin river are below 350000 people. Thus in the rapid development of Lintong county tourism, there also are some problems, including tourist attractions tourist reception number bear gap is too large, shadow effect obviously greatly reduces the development velocity of Lintong county tourism. It is imminent to solve the problem of shadow effect in tourism development.

\section{Shadow Effect Analysis of Qin Shihuang Terracotta Warriors Museum and Banpo Ruins} Museum on Bian Que Tomb, Lin Xiangru Tomb, Jiang Village Cite

\section{A. The comparative analysis of shadow area and shadowed area resources}

a. Qinshihuang terracotta warriors, Bian Que tomb, and Lin Xiangru tomb tourism resources comparative analysis

Bian Que tomb, Lin xiangru's tomb, and Qinshihuang terracotta warriors are mausoleum and tomb tourism resources, but there is a big gap between their level of scenic area, scale, historical and cultural connotation mining, etc.: 
First, Qinshihuang terracotta warriors are one of the world cultural heritages, national AAAAA scenic spot, and national museum with very high scenic area level and visibility. Although Bian Que tomb and Lin Xiangru tomb have a certain level and awareness, there is a considerable difference in comparison with Qinshihuang terracotta warriors.

Second, Qinshihuang terracotta warriors are a relatively developed, mature and perfect tourism scenic area with profound historical cultural connotation. Bian Que tomb and Lin Xiangru tomb lack connotation development in scenic area development and construction with a lack of attracting tourists to visit the stunt.

Finally, there is a gap in scenic spot on the replication. Bian Que tombs, and Lin Xiangru tomb besides Lintong, still are developed in other provinces and cities. The specific location is still controversial, and Qinshihuang terracotta warriors have its unique tourism resources, so it is difficult to simulate and replicate.

Although Bian Que tomb, Lin Xiangru tombs have a gap comparing with terracotta warriors in many ways, still they have their own characteristics and advantages. Bian Que tomb is built relying on Bian Que tomb, but it is still a memorial hall, which shows many medicines and medical knowledge, famous medical ones introduction such as Bian Que. Its unique medical resources make it a big advantage. Imperial mausoleum has been very famous tourism resource in Shanxi province, and its appeal to tourists is self-evident, but captains' tombs are still worth a visit. Lin Xiangru is known for his smart, courage, and measure in the history, so his tomb is still of a certain appeal.

b. Comparative analysis on Banpo museum and Jiang village site tourism resources

Banpo museum and Jiang village site are neolithic cultural sites. The two have similarity and dissimilarity. On scenic spot size and grade, Xian Banpo museum is international AAAA level scenic spot. In addition, Banpo site is published by State Council as one of the first batch of national key cultural relic's protection unit, and in May 2008, State Administration of Cultural Heritage rated it "national museum". And although Jiang village site is a national key cultural relic's protection unit, there still exists certain gaps compared with Banpo.

In historical and cultural value of scenic spots, Banpo site collects all kinds of 18000 pieces of cultural relics, including 4000 pieces of cultural relics above 3level, specimens of more than 300 pieces, and there are a number of human and animal bones specimens. Banpo relic shows people more than six thousand years ago Banpo people's living environment, architectural form, sacrifices cite production technology, burial customs, etc. with historical heritage sites and a large number of physical articles. And Jiang village site contains province phase ii type and v type neolithic Yangshao culture's Banpo type, type of historians, temple bottom ditch type, Xiwang Village type and Longshan culture and so on culture remains. Cultural relics unearthed are ten thousand pieces, which is preserved, and the layout is unprecedented clear, and has important scientific meaning and historical and cultural value.

\section{B. Bian Que tomb, Lin Xiangru tomb, Jiang village site shadow forming reason analysis}

a. Tourist consumption preference

First, in terms of tourist consumption psychology, according to the spatial behaviors of tourists, tourists travel money, time, energy are limited, so tourists will choose higher level tourist attractions. And, in order to achieve benefit maximization, they go to higher level scenic spots, and often do not stay to visit low level tourist attractions, but continue to go to other high tourist level sites. According to the above analysis, Bian Que tomb, Lin Xiangru tomb, Jiang village site are of lower scenic spot level than Qinshihuang terracotta warriors and Banpo site, and this is why a lot of tourists choose not to visit Bian Que tomb, Lin Xiangru tomb, Jiang village site.

At the same time, decision making in tourism enterprises also largely causes the shadows formation and intensification of Bian Que tomb, Lin Xiangru tomb, Jiang village site. Through the network retrieval of east of xian tour line, one day tour is given priority to, and one day trip destination is most commonly Terra Cotta Warriors; the second is Mount Li HuaQing pool scenic area, followed by Banpo museum. Because tourists stay within a day, the route does not contain Bian Que tomb, Lin Xiangru tomb, and Jiang village sites.

b. The insufficient development and not enough propaganda of scenic spots 
Although Bian Que tomb, Lin Xiangru tomb, Jiang village site are slightly lower in scenic spot level than Qin terra-cotta warriors and Banpo site, they also have their own tourism features, and their tourism resources have certain heterogeneity with high development value and monopoly. However, because of Xian Lintong's society and economy development level, tourism resources development level is low. With not well organized special tourism products, Bian Que tomb, Lin Xiangru tomb, Jiang village site travel are always in the "shadow" of Terra Cotta Warriors and Banpo relics' tourism.

In addition, in scenic spot propaganda, Bian Que tomb, Lin Xiangru tomb, Jiang village site are mainly through network advertisement publicity, and outdoor advertising and self-administration advertising are relatively scarce. All the advertisement publicity is relatively small. In sales promotion and public relations, scenic spot publicity is little also, and lack scenic spot information even in tourism website.

c. Scenic spot has no clear image

Currently, Bian Que tomb, Lin Xiangru tomb, Jiang village site construction quality is not good with no deep historical culture mining. Scenic spot function is not clear and has no distinct image slogan and characteristic image identification, so tourists are difficult to touch the essence of Bian Que tomb, Lin Xiangru tomb, Jiang village sites. Bian Que tomb, Lin Xiangru tomb, Jiang village sites are scenic spots which have not yet formed distinctive tourism scenic features in visitors mind.

d. Geographical reasons

Though there is highway geographical location condition for Bian Que tomb, Lin Xiangru tomb, Jiang village site, it is more convenient for driving tourists, however, the main bus lines are not through the sites, which to a certain extent affects convenience, so there are some disadvantages.

\section{Bian Que Tomb, Lin Xiangru Tomb, Jiang Village Site Breakthrough Shadow Effect Strategy}

The main reason scenic spots are in shadow area is that their own tourism resources meet higher level, larger tourism scenic spot, therefore strengthen the construction of scenic spot, play its unique advantages of tourism resources, avoid high level scenic spot, turn cover into the shadows of superposition and cooperate in the breakthrough are critical in shadow area. Combining home and abroad shadow area scenic development countermeasures and three big scenic spot actual situation: Bian Que tomb, Lin Xiangru tomb, Jiang village site, concrete strategies are proposed for the construction and development of scenic spot.

\section{A. Bian Que tomb's medicine meal law}

In recent years, people increasingly focus on food safety and health, traditional Chinese medicine keeping in good health food and beverage are in fashion. Bian Que tomb is not only a good place for tourists to commemorate Bian Que, and shows rare medicinal herbs and traditional Chinese medicine healer biography with outstanding contribution. Traditional Chinese medicine has very deep origin, promotes that medicine is inferior to eat the right food, and famous doctor Bian Que is also a doctor advocating prevention is better than cure. Take this as background, launch different season good health food and beverage of traditional Chinese medicine, pay attention to join often used drugs, such as: Chinese wolfberry, radix polygoni multiflori, radix paeoniae alba, rhizoma ligustici wallichii, ginseng, astragalus, and so on. This can fill the gaps in Lintong tourism market characteristic catering, and tourism impetus to the development of peripheral industries; second, it can enrich tourism Bian Que tomb content, create a propaganda window, and break through Bian Que tomb shadow effect.

\section{B. Lin Xiangru tomb avoiding hot spots way}

Xian is very rich in tourism resources, especially mausoleum kind tourism resources, including Qinshihuang tomb, Qian tomb, Mao tomb and other well-known tourist attractions, which attract a large number of tourists to visit every year. Through analysis, it can be found that they are all royal tombs with grand scale building. There are a large number of precious cultural relics with profound historical and cultural connotation. Lin Xiangru tomb does have a larger gap in these areas. To break through the shadow effect, it can only choose to avoid Qinshihuang, Qian tomb, Mao tomb mausoleum tourist hot spots, and dig its unique advantage of tourism. 
Lin Xiangru tomb can make use of the advantages of the following two points to break through shadow effect: on the one hand, loyal captains' tombs are different from royal tombs with different architectural style and ritual. By visiting Lin Xiangru tomb, people can have wider knowledge of ancient tomb art; on the other hand, in face of a lot of tourists eager to travel and deter crowded situation, Lin Xiangru tomb as immature tourist attraction, with less visitor numbers, good scenic spot order, it can become a great place to relax for tourists.

To make full use of the advantages, specific operation should pay attention to propaganda to enlarge popularity, establish official website, and post tourism ads on the website; second, pay attention to regulation environment of scenic spot. Lin Xiangru tomb covers a large area, but the surrounding environment is relatively poor, and messy. In recent construction, pay attention to sort out relative value of larger part of building. In addition, Lin Xiangru tomb also should be conducted corresponding development and perfection on traffic location.

\section{Jiang village sites and Banpo tourism hand in hand cooperation}

To breakthrough Jiang village site shadow effect, improve the image of scenic area by strengthening regional cooperation, hand in hand with Banpo ruins museum, complement resource advantages, and use tourism cooperation method.

Regional combination status of tourism products have been also the important measure of scenic spots quality. Jiang village sites cooperation with Banpo site can make tourists have a more comprehensive understanding of neolithic culture,include Yangshao culture and longshan culture two stages, which form a relatively complete system, and the two complement each other in value. In order to make the cooperation continued to deepen, establish a good foundation of cooperation, promote tourism development, Lintong tourism should be led by government, with competent department productive, multi-sectoral cooperation to form a special tourism development coordination committee, with members led by Jiang village sites and Banpo sites, in order to ensure the efficient and authority of the committee. The specific practices are as follows:

First of all, integrate the two scenic area tourist resources and its characteristics, form government's leading tourism development, design tourism slogan, logo and propaganda materials together to change the original mode of both brochure scenic spots. Jiang village sites can also be combined with Banpo registered face water-wave basin trademark to develop tourism logo design itself, and compensate for Jiang village site tourism logo and souvenirs. Fish is taken as the totem of neolithic age. In Jiang village site, a fish bird patterns gourd bottle also unearthed with model modeling, and beautiful pattern. Jiang village site can use the pattern design on the bottle for tourism logo, and it would be beautiful and unique.

Second, design unified design tourism routes. Plan direct route between the two scenic spots tourism lines, with direct free shuttle buses, and bargain Jiang village site tickets holding Banpo site scenic spot ticket to expand propaganda, and attract tourists. The combination of the two match forms superposition effect. Reach the results of one plus one is greater than two, and builds the overall advantage and competitiveness of regional tourist products.

Finally, the scenic spot can also develop new tourist souvenirs. Jiang village site unearthed many novel and attractive pottery products, such as fish bird patterns gourd bottle, etc., which is now still a unique style of decoration. The scenic spot shall make fish bird patterns gourd bottle imitation as main products to improve the participation of tourists and memorable product, and can let visitors make them personally, and coloring. In addition, pay attention to the diversity of souvenirs. Pottery products will have certain restriction, so you can put fish bird tattoo, the neolithic totem pattern on scarf, or be printed on the small block in front of beret as decoration, which is chic and beautiful.

\section{Conclusion}

All in all, Bian Que tomb, three big scenic spots Lin Xiangru tomb, Jiang village site's breakthrough shadow effect should pay attention to scenic spot construction and assistance from external power to drive the combination. In this paper, we proposed the compensate method and hot spots avoiding method and tourist cooperation method to play a role of Bian Que tomb, Lin Xiangru tomb and Jiang village site breakthrough shadow effects. 


\section{References}

[1] Wang Yanyong. Mencius home tourism development strategy research [J]. Journal of Geography and Research, 1995, I 5, (2)

[2] Xu Chunxiao. Tourism area moderate development and optimal case study [J]. Journal of Economic Geography, 1995 (2)

[3] Xu Chunxiao. Destination shielding theory study [J]. Journal of Tropical Geography, 2001, 21 (1)

[4] Xu Chunxiao. 21st century new field of China tourism geography: tourism zone optimal study [J]. Journal of Travel, 2000, (1)

[5] Li Xuesong. Tourism image block: basic properties and space performance [J]. Journal of Ideological Front, 2010, 4 (2)

[6] Yu Fei, Xu Yangyang. The formation and evolution of tourism scenic spot image block research [J]. Journal of Anhui Agricultural University, Social Sciences, 2011 (5)

[7] Yu Fei. Domestic research review on tourism destination image block theory [J]. Market Modernization, 2009, (18)

[8] Yang Zhenzhi, Chen Jin. "Cover image" and "image superposition" theory and empirical research [J]. Journal of Travel, 2005, (03)

[9] Su Lijuan, Yan Shang. The optimal development countermeasure of tourism resources in a blocking condition - in Hebei city for example [J], Development, 2008 (3)

[10] Li Lingling. Heilongjiang ice and snow tourism image masking effect research [D]. Harbin: Heilongjiang University, 2012

[11] Guo Yanfang. "Shadow" urban tourism image research based on tourist cognition [D]. Xian: Shanxi Normal University, 2011

[12] Hughes, H. and Allen,D.Culturaltourismin Central and Eastern Europe: the views of induced image formationagents[J]. Tourism Management, 2005, 26(2): 173-183

[13] Hunt ,JD. Image-A Factor in Tourism. Unpublished doctor all dissertation [D]. Colorado State University, 1971.

[14] Jiang Kunfu, Zhang Shulin, Ge Xiaotao, Tang Weiliang. Weak tourism strategy research in spatial competition----taking an example of Chongqing Bishan [J]. Journal of Lanzhou Institute of Education, 2010, 26, (2) : 61-68.

[15] Ye Meijun. Tourism theory and case study [D]. Xiamen: Xiamen University, 2005.

[16] Zhuang Meihua. On "Wuyi" tourism breakthrough shadow effect research [J]. Journal of huaihai Institute of Technology, 2011, 9 (21)

[17] Ren Jing. Location optimal tourism destination development strategy research - taking Rizhao city in Shandong province for example [D]. Beijing: Northern Traffic University, 2007.

[18] Wang Juan. Tourist non-optimal tourism research - taking Hami district for example [D]. Urumqi: Xinjiang Normal University, 2007.

[19] Fan Yuxian. Lushan mountain Jiujiang city tourism shadow effect analysis and countermeasure research [J]. Journal of Wuhan Vocational and Technical College, 2009, 8 (5)

[20] Mao Weidong, Huang Zhen-fang. Borderline tour city tourism development exploration----taking Lianyungang city for example [J]. Journal of Human Geography, 2008 (6)

Project: Qinling ecological tourism sustainable development research under the background of Xian tourism city construction, 2013 Xian science and technology bureau soft science project, numbering SF1312-1; 